University of Wollongong

Research Online

Faculty of Engineering and Information

Faculty of Engineering and Information

Sciences - Papers: Part A

Sciences

$1-1-2007$

\title{
Fast encodable and decodable irregular repeat accumulate codes from circulant permutation matrices
}

S Tong

Xidian University, sheng@uow.edu.au

S Zhang

Xidian University

B Bai

Xidian University

X Wang

Xidian University

Follow this and additional works at: https://ro.uow.edu.au/eispapers

Part of the Engineering Commons, and the Science and Technology Studies Commons

Research Online is the open access institutional repository for the University of Wollongong. For further information contact the UOW Library: research-pubs@uow.edu.au 


\title{
Fast encodable and decodable irregular repeat accumulate codes from circulant permutation matrices
}

\author{
Abstract \\ An approach to construct irregular repeat accumulate (RA) codes from circulant permutation matrices is \\ presented. The constructed irregular RA codes can be encoded with shift registers and decoded in the \\ turbo decoding fashion, which leads to a much faster convergence rate compared to the conventional full \\ parallel decoding fashion.

\section{Keywords} \\ repeat, irregular, decodable, encodable, accumulate, fast, matrices, codes, permutation, circulant \\ Disciplines \\ Engineering | Science and Technology Studies

\section{Publication Details} \\ S. Tong, S. Zhang, B. Bai \& X. Wang, "Fast encodable and decodable irregular repeat accumulate codes \\ from circulant permutation matrices," Electronics Letters, vol. 43, (1) pp. 48-49, 2007.
}




\section{Fast encodable and decodable irregular repeat accumulate codes from circulant permutation matrices}

\section{S. Tong, S. Zhang, B. Bai and X. Wang}

An approach to construct irregular repeat accumulate (RA) codes from circulant permutation matrices is presented. The constructed irregular RA codes can be encoded with shift registers and decoded in the turbo decoding fashion, which leads to a much faster convergence rate compared to the conventional full parallel decoding fashion.

Introduction: Because of their exceptional performance and low decoding complexity, low-density parity-check (LDPC) codes [1] have received much interest in the past decade. Many efforts have been made regarding their theoretical analysis and practical implementation issues. However, there are still two deficiencies in practical applications of LDPC codes, i.e. high encoding complexity and relative slow convergence rate compared to turbo codes. In fact, there are already some special classes of LDPC codes, which admit fast encoding procedures such as repeat-accumulate (RA) codes. Moreover, our previous work shows that Gallager codes [1] can be decoded in the turbo decoding fashion using a message passing schedule [2], dubbed turbo decoding schedule (TDS), which shows a faster convergence rate than the conventional flooding schedule (FS) [3]. Here, we build the parity check matrices using circulant permutation matrices for the irregular RA codes [4] to allow for TDS and thus accelerate the convergence rate. The resulting irregular RA codes just like Gallager's regular LDPC codes can also be interpreted as intersected codes and naturally decoded using TDS.

Codes from circulant permutation matrices: Regular LDPC codes from circulant permutation matrices have been investigated by many (see, e.g. [5]) and the references therein. Here, we extend the regular codes to irregular ones by introducing zero square matrices. Following the notations in [5], a $z \times z$ circulant permutation matrix is determined by a cyclic-shift parameter $p$, which indicates that the only ' 1 ' of the $r$ th row is located at the $(r+p) \bmod z$ th position $(0 \leq r \leq z-1)$. Here, the parity check matrix for an irregular RA code is given by $\mathbf{H}=$ $\left[\left(\mathbf{H}_{1}\right)_{M z \times(N-M) z} \mid\left(\mathbf{H}_{2}\right)_{M z \times M z}\right]$, where $\mathbf{H}_{1}$ and $\mathbf{H}_{2}$ correspond to the systematic bits and parity bits, respectively, and consist of circulant permutation matrices and zero square matrices. It is known that for irregular RA codes $\mathbf{H}_{2}$ is of zigzag pattern. Here, we build a zigzag equivalent form for $\mathbf{H}_{2}$ as follows: place $z \times z$ identity matrices along the diagonal and sub-digonal and a circulant matrix with $p=-1$ on the upper rightmost corner, in which the ' 1 ' in the last column is deleted; the remaining sub-matrices are chosen to be zero square matrices. Fig. 1 shows a simple example along with the shift register encoder From Fig. 1, it is seen that the irregular RA codes can be viewed as an intersection of three super codes, each defined by a parity check matrix formed via a block of circulant permutation matrices in $\mathbf{H}$. Hence, TDS can be readily applied in the decoding of this code. The basic idea of TDS is to decode an intersected code in the turbo decoding fashion by employing a soft-in soft-out decoder for each super code and exchanging extrinsic information between them [3]. Note that all the parity check relations within each super code are independent and thus can be decoded simultaneously, which implies that TDS for the irregular RA codes can be performed in a partial parallel fashion.

Code design procedure: An irregular LDPC code ensemble could be specified by a node degree distribution pair $(\Lambda, P)$ with generating functions $\Lambda(x)=\sum_{i=2}^{d_{l}} \Lambda_{i} x^{i}$ and $P(x)=\sum_{j=2}^{d_{r}} P_{j} x^{j}$, where $\Lambda_{i}$ is the fraction of variable nodes of degree- $i, P_{j}$ is the fraction of check nodes of degree- $j, d_{l}$ is the maximum variable node degree, and $d_{r}$ is the maximum check node degree. It is easy to check that the following constraints hold.

$$
\left\{\begin{array}{l}
\Lambda(1)=1, P(1)=1 \\
\sum_{i=2}^{d_{l}} i \Lambda_{i}=(1-R) \sum_{j=2}^{d_{r}} j P_{j}
\end{array}\right.
$$

where $R$ is the code rate. Here, we constrain $\Lambda_{2}=M / N$ and all the degree-2 variable nodes to be parity check bits. Moreover, $P(x)$ is restricted to have a concentrated form, i.e. $P(x)=(1-P) x^{d_{r}-1}+P x^{d_{r}}$
[6]. Hence, the free dimension of the vector formed by the node degree distribution pair $(\Lambda, P)$ is $L=d_{l}-3$. Denote as $p=\left(\Lambda_{4}, \ldots, \Lambda_{d_{l}^{-1}}, P\right)$ the $L$-dimensional vector.
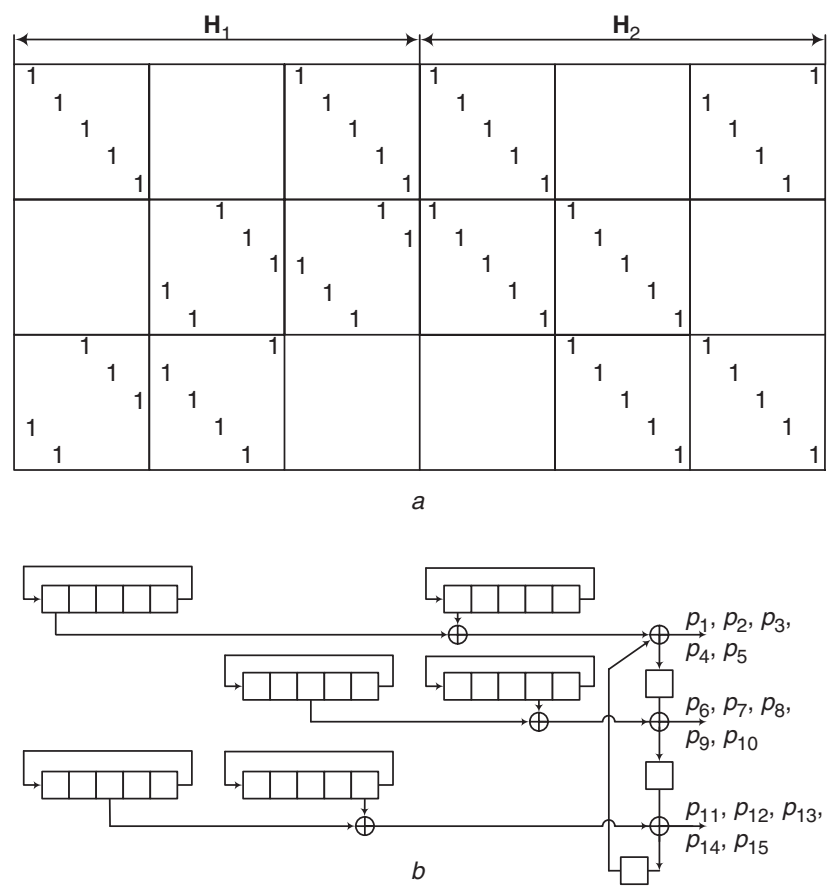

Fig. 1 Rate-1/2 irregular $R A$ code from circulant permutation matrices $a$ Parity check matrix (shadowed ' 1 ' in last column deleted)

$b$ Shift-register encoder $\left(p_{i}\right.$ denotes $i$ th parity check bit corresponding to $i$ th column of $\mathbf{H}_{2}$ )

Using (1) and $\Lambda_{2}=M / N$ to solve for $\Lambda_{3}$ and $\Lambda_{d_{l}}$ gives

$$
\left\{\begin{array}{l}
\Lambda_{d_{l}}=\frac{(1-R)\left(d_{r}+P\right)-\sum_{i=4}^{d_{l}-1} \Lambda_{i}(i-3)-3}{d_{l}-3} \\
\Lambda_{3}=R-\sum_{i=4}^{d_{l}} \Lambda_{i}
\end{array}\right.
$$

The code design procedure is as follows.

1. Choose proper $M, N$, and $z$ according to the code length, rate, $d_{l}$, and $d_{r}$. 2. Use (2) and the Gaussian approximation of density evolution [6] to generate a node degree distribution pair $(\Lambda, P)$ optimised for threshold. Note that since $\Lambda_{i}\left(\right.$ resp. $\left.P_{j}\right)$ is a multiple of $1 / N($ resp. $1 / M)$ and $N$ and $M$ are small integers (less than 100), optimisation methods are not necessary. Randomly producing sufficient distribution pairs (say 10L) suffices to obtain a good one.

3. Construct a binary $M \times N$ base matrix $\mathbf{H}_{b}$ according to the distribution pair $(\Lambda, P)$ using PEG algorithm [7] for a large girth.

4. Replace each ' 1 ' (resp. ' 0 ') in $\mathbf{H}_{b}$ with a circulant matrix (resp. a zero square matrix), the cyclic-shift parameter of which can be generated using Theorem 2.1 in [4] to avoid short cycles.

Example and simulations: Using the above design procedure, a rate-1/2 and length-1000 irregular RA code has been constructed with $M=10, N=20$, and $(\Lambda, P)$ given by

$$
\left\{\begin{array}{l}
\Lambda(x)=0.5 x^{2}+0.3 x^{3}+0.2 x^{7} \\
P(x)=0.4 x^{6}+0.6 x^{7}
\end{array}\right.
$$

For simplicity, in (3) we treat the degree-1 variable node for the last column of $\mathbf{H}$ as degree-2. Interestingly, it is found that this 'discretised' degree distribution pair exhibits almost the same threshold as the optimal edge degree distribution for rate- $1 / 2$ with $d_{l}=d_{r}=7$ in [6, p. 183]. This implies that by choosing proper $M$ and $N$ matching to $d_{l}$ and $d_{r}$ the threshold of an optimised 'discretised' node degree distribution pair would approach to that of an optimised 'continuous' one. Moreover, 'discretised' node degree distribution pairs can be generated without the aid of optimisation methods and are more suitable for hardware implementation. 
Fig. 2 depicts the bit error rate (BER) performance of the above code using the sum-product algorithm under FS and TDS. To our best knowledge, this code performs similar to the best LDPC codes with the same rate and code length. However, it has a good algebraic structure and thus is suitable for hardware implementation. Similar to the case of Gallager codes, this irregular RA code shows a much faster decoding convergence rate using TDS than FS: 20 iterations are enough for TDS to achieve the BER performance at 100 iterations when using FS.

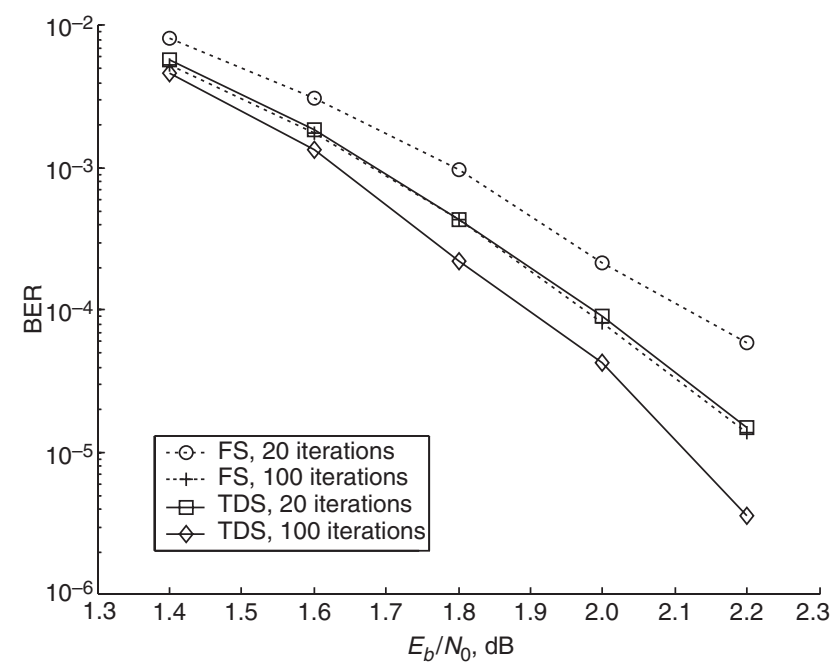

Fig. 2 BER performance of rate-1/2 and length-1000 irregular RA code from circulant permutation matrices under flooding schedule (FS) and turbo decoding schedule (TDS) on AWGN channel

Conclusion: An approach to construct parity check matrices of irregular RA codes from circulant permutation matrices is presented. Codes constructed by this approach allow for a fast encoding procedure using shift registers and can be decoded under TDS, thus leading to a fast convergence rate.

Acknowledgment: This work was supported by NSFC grants 6049316 and 60472098 .

(C) The Institution of Engineering and Technology 2007

14 October 2006

Electronics Letters online no: 20073184

doi: $10.1049 / \mathrm{el}: 20073184$

S. Tong, S. Zhang, B. Bai and X. Wang (State Key Lab of ISN, Xidian University, People's Republic of China)

E-mail: ts_xd@163.com

S. Zhang: Now with the Fuzhou RockChip Semiconductor Co. Ltd, People's Republic of China

\section{References}

1 Gallager, R.G.: 'Low-density parity-check codes' (MIT Press, Cambridge MA, 1963)

2 Kschischang, F.R., Frey, B.J., and Loeliger, H.-A.: 'Factor graphs and the sum-product algorithm', IEEE Trans. Inf. Theory, 2001, 47, (2), pp. $498-519$

3 Tong, S., and Wang, X.: 'A simple convergence comparison of Gallager codes under two message-passing schedules', IEEE Commun. Lett., 2005, 9, (3), pp. 249-251

4 Jin, H., Khandekar, A., and McEliece, R.: 'Irregular repeat-accumulate codes'. Proc. 2nd Int. Symp. on Turbo Codes and Related Topics, 2000, pp. $1-8$

5 Fossorier, M.P.C.: 'Quasi-cyclic low-density parity-check codes from circulant permutation matrices', IEEE Trans. Inf. Theory, 2004, 50, (8), pp. $1788-1793$

6 Chung, S.Y.: 'On the construction of some capacity-approaching coding schemes'. PhD Dissertation, Department of Electrical Engineering, Massachusetts Institute of Technology, Cambridge, MA, 2000

7 Hu, X.-Y., Eleftheriou, E., and Arnold, D.-M.: 'Progressive edge-growth Tanner graphs'. Proc. IEEE GLOBECOM Conf., 2001, pp. 995-1001 